\title{
The current classification of urothelial neoplasms
}

\author{
David J Grignon \\ Department of Pathology and Laboratory Medicine, Clarian Health, Indiana University School of Medicine, \\ Indianapolis, IN, USA
}

\begin{abstract}
The classification of urothelial neoplasms has been a subject of significant controversy and debate over the last decade. Only recently has a general level of agreement developed on the utility of the classification first proposed by the World Health Organization and the International Society of Urologic Pathology in 1998. Recent adoption of this scheme in therapeutic guidelines indicates the clinical utility of the system. In this review a brief historical perspective is presented, followed by a review of the classification system, the histological criteria for the specific categories and the clinical significance of these diagnoses.

Modern Pathology (2009) 22, S60-S69; doi:10.1038/modpathol.2008.235
\end{abstract}

Keywords: urothelial neoplasms; classification; grading; prognosis; treatment

The last decade has seen a tremendous upheaval in the very important area of urothelial neoplasm classification, which only recently seems to have settled down to a level of general concurrence. For over two decades, the World Health Organization (WHO) classification and grading of urothelial neoplasms ${ }^{1}$ dominated, although several variations and different schemes were published. In the early 1990s, several factors emerged that resulted in the need to reevaluate this approach. First, the controversy of calling grade 1 papillary tumors 'carcinoma' arose with several groups led by Dr William Murphy beginning to call all tumors in the lowgrade end papilloma. ${ }^{2,3}$ Second, the use of intravesical therapy as a standard practice in the treatment of high-risk non-invasive papillary tumors demanded that high-risk tumors be clearly identified. Third, the WHO (1973) system was criticized for the imprecision of the published criteria (Table 1), leading many pathologists to essentially use this three-grade system to create five grade groups $(1,1-2,2,2-3$ and 3$)$; one result being that only a small percentage of cases were placed in the grade 3 group. For example, in a review of three clinical studies, only 25 of $280(8.9 \%)$ newly diagnosed noninvasive papillary tumors (pTa) were called grade $3 .^{4}$ The effect of the latter was confusion among

Correspondence: Dr DJ Grignon, MD, Clarian Pathology Laboratory, 350 West Eleventh Street, Room 6014, Indianapolis, IN 46202, USA.

E-mail: dgrignon@iupui.edu

Received 16 December 2008; accepted 16 December 2008 clinicians as to how to treat grade 2 pTa tumors, a category that clearly included many high-risk patients who could benefit from intravesical therapy as well as many patients with low-risk disease for whom intravesical therapy may not be appropriate or necessary. Multiple studies have demonstrated that this group includes high-risk patients with progression to invasive carcinoma reported in up to $20 \%$ of patients and cancer-specific death in $13-20 \% .{ }^{5,6}$

Recognizing the many emerging issues, in 1997 Dr FK Mostofi organized a meeting of a small group of urologic pathologists, urologists and urologic oncologists to address these concerns. This was followed by a much larger consensus conference that was held under the auspices of the International Society of Urologic Pathology (ISUP) in March of 1998. The results of this consensus were adopted by the WHO and the results were published in 1998 as the WHO/ISUP consensus classification. ${ }^{7}$ Most controversial was the adoption of the term papillary urothelial neoplasm of low malignant potential. This represented a compromise term where the papilloma and carcinoma advocates could be comfortable and allowed that controversy to be brought to resolution. Most important was the adoption of the grading system that had been described by Malmström et al. ${ }^{8}$ The value of the latter was viewed as twofold; first, the morphologic criteria for applying the scheme were well defined and second, it appeared to place the majority of patients with high-risk disease into the high-grade category. 
Table 11973 WHO grading criteria ${ }^{1}$

Grade 1 Tumors with the least degree of cellular anaplasia compatible with a diagnosis of malignancy Histologic features between grades 1 and 3

Grade 2

Tumors with the most severe degrees of cellular anaplasia

Table 22004 WHO/1998 ISUP classification

Normal

Hyperplasias

Flat lesions with atypia

Reactive (inflammatory) atypia

Atypia of unknown significance

Dysplasia (low-grade intra-urothelial neoplasia)

Carcinoma in situ (high-grade intra-urothelial neoplasia)

Papillary neoplasms

Papilloma

Inverted papilloma

Papillary urothelial neoplasm of low malignant potential

Papillary carcinoma, low grade

Papillary carcinoma, high grade

Invasive neoplasms

The publication of the 1999 WHO blue book the following year introduced a variation on this system with the splitting of the low- and high-grade categories of the $1998 \mathrm{WHO} / \mathrm{ISUP}$ classification into three groups (grades 1,2 and 3) while retaining the papillary urothelial neoplasm of low malignant potential category. ${ }^{9}$ This re-ignited the controversy with some experts urging a return to the 1973 WHO grading system. ${ }^{10}$ Others criticized the $1998 \mathrm{WHO} /$ ISUP system as simply representing a renaming of the $1973 \mathrm{WHO},{ }^{11}$ a clearly incorrect interpretation. ${ }^{12}$ At a subsequent meeting in Ancona, Italy (2001) a modified version of the 1973 WHO was proposed. ${ }^{12}$

This issue became the primary focus of discussion at the WHO meetings prior to the release of the 2004 WHO classification. Following extensive debate and discussion, it was agreed overwhelmingly to essentially reproduce the 1998 WHO/ISUP classification as the 2004 WHO recommended classification scheme (Table 2). ${ }^{13}$ Similarly the authors of the 4 th Series Armed Forces Institute of Pathology fascicle covering bladder neoplasia have followed this system. ${ }^{14}$ With this consistent approach adopted by arguably the two most influential references for tumor classification and grading, work can continue on evaluating the biologic and clinical relevance and value of this system. ${ }^{15}$ The success of this in addressing the key issues outlined in the first paragraph has been stressed, in particular the reclassification of high-risk grade 2 tumors (WHO, 1973) in the high-grade papillary carcinoma category. ${ }^{12,16,17}$ For example, in the study by Yin and Leong, ${ }^{17} 13$ of 46 WHO (1973) grade 2 tumors (28\%) were placed in the WHO (2004) high-grade category resulting in $23 \%$ of all cases being considered high grade in WHO 2004 compared with only $4 \%$ being called grade 3 in the 1973 WHO system. Similarly, Samaratunga et al, ${ }^{16}$ reviewed 134 papillary tumors of which 6 (4\%) had been reported as grade 3 (WHO, 1973); on review, they considered $29(22 \%)$ to be high grade by WHO/ISUP 1998.

This approach has now been embraced by many urologic oncologists with interest in bladder cancer. It has important application in the contemporary treatment of pTa tumors. The reclassification of high-risk grade 2 tumors (WHO, 1973) into the highgrade category (WHO, 2004) has resulted in a large and better defined group of patients with low-risk pTa tumors. In a series of 215 patients with lowgrade (papilloma, papillary urothelial neoplasm of low malignant potential and low-grade papillary carcinoma) pTa tumors from the Memorial Sloan Kettering Cancer Center treated by transurethral resection only, progression to high-grade pTa or invasive (pT1) carcinoma occurred in only 3 and $5 \%$ of patients, respectively, with a median follow-up of 8 years. ${ }^{18}$ A recent prospective study has lent further support to these findings. ${ }^{19}$ This experience has led to the suggestion that patients with low-grade pTa tumors can be followed less frequently. ${ }^{20}$ Similarly, Nieder and Soloway ${ }^{21}$ currently treat patients with papilloma and papillary urothelial neoplasm of low malignant potential by transurethral resection alone, low-grade pTa tumors by transurethral resection with a single dose of mitomycin $\mathrm{C}$ and high-grade pTa tumors by transurethral resection with mitomycin $\mathrm{C}$ and bacillus Calmette-Guérin immunotherapy. In December 2007, the American Urological Association reinforced the current approach in its guidelines for the treatment of non-muscle invasive bladder cancers by separating non-invasive papillary tumors into two groups-low grade and high grade ${ }^{22}$ - with different treatment recommendations for each group. The College of American Pathologists also utilizes WHO 2004 in its recommendations for the reporting of urothelial tumors. ${ }^{23}$

\section{Flat lesions}

\section{Hyperplasia}

Historically, the term 'hyperplasia' has been equated with counting cell layers and specifically considering the epithelium to be hyperplastic if there were more than seven cell layers. It is well recognized that the apparent number of cell layers in the normal urothelium is variable and dependent on the state of contraction of the bladder wall. The current classification recognizes hyperplasia as when there is a 'markedly thickened mucosa without atypia' (Figure 1). Counting cell layers is not recommended. There is no significant cytologic or architectural atypia. Hyperplasia can have a pseudopapillary architecture. Hyperplasia is usually part of 


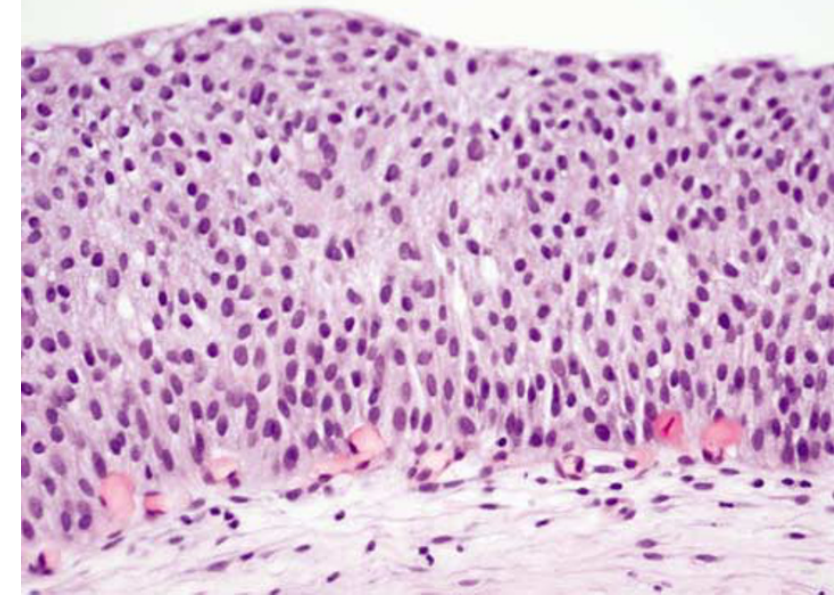

Figure 1 Urothelial hyperplasia. The urothelium is markedly thickened without significant architectural or cytologic abnormalities.

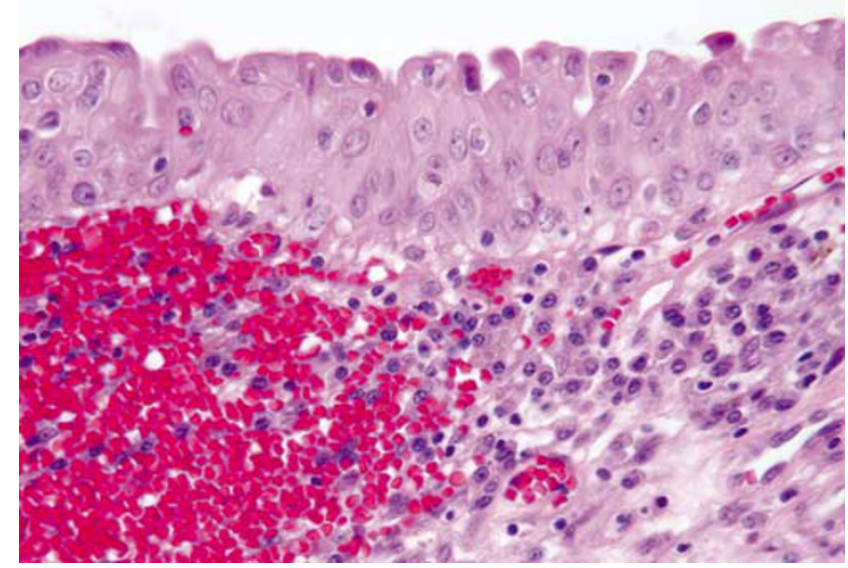

Figure 2 Reactive urothelial atypia. The nuclei are mildly enlarged, rounded and have small nucleoli. Note the intense chronic inflammatory infiltrate in the lamina propria.

a reactive process in response to inflammation or other causes of irritation. The relationship between hyperplasia and neoplasia is unknown.

\section{Reactive Atypia}

In the presence of acute and/or chronic inflammation, the urothelium shows a wide range of reactive changes. There is usually a history of instrumentation, infection or treatment with intravesical agents. Some patterns of atypia are associated with specific etiologies. In reactive atypia, the epithelium may or may not be thickened. Although a thickened epithelium is typically associated with a reactive process, carcinoma in situ can also produce a thicker than normal epithelium. Nuclei are uniformly enlarged, vesicular and may have prominent usually centrally located nucleoli (Figure 2).

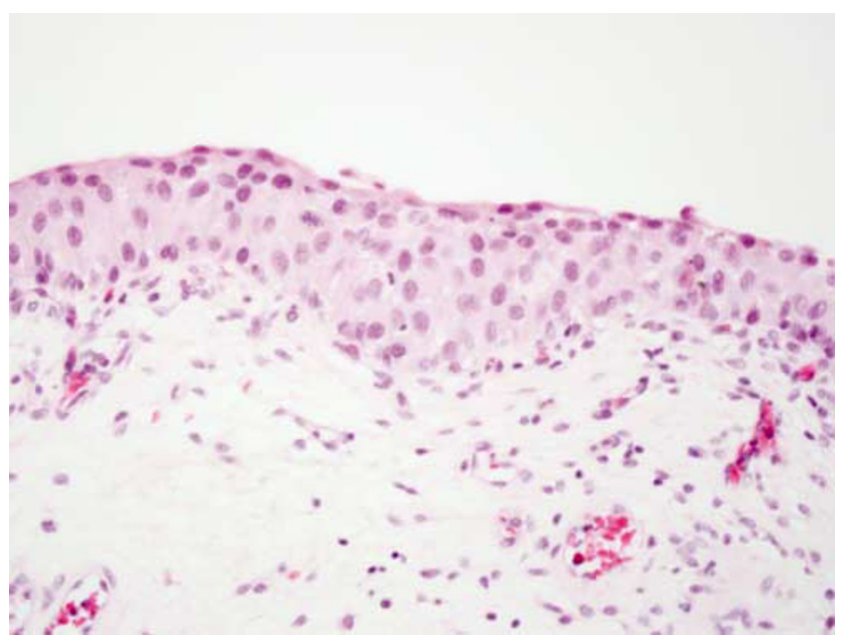

Figure 3 Urothelial dysplasia. There is mild nuclear enlargement with a somewhat haphazard distribution of nuclei within the urothelium. Note the mitotic figure in the upper level of the urothelium.

Mitoses can be frequent and are located in the lower epithelial layers. Inflammation is almost always present.

\section{Atypia of Unknown Significance}

One of the major gray zones in any consideration of intraepithelial lesions is between reactive atypia and true neoplastic (dysplastic) alterations. Reproducibility studies have clearly demonstrated the lack of consistency in this particular area. This category was created to include those instances where a lesion cannot be confidently placed in the reactive versus dysplastic categories. Histologically, there is usually an inflammatory background. The degree of cytologic atypia is judged to be outside of the accepted range for reactive processes, although this possibility cannot be excluded. Reevaluation after inflammation subsides may resolve the problem, particularly in the follow-up of patients with known urothelial neoplasia who have been treated with intravesical therapy. In one study, none of 35 patients diagnosed with atypia of unknown significance developed urothelial neoplasia with a median 3.5 years of follow-up. ${ }^{24}$

\section{Dysplasia (Low-grade Intra-Urothelial Neoplasia)}

This category also suffers from a significant problem in diagnostic reproducibility. The natural history of lesions with dysplastic features of a lesser degree than the moderate to severe categories is unknown. ${ }^{25}$ There is however some evidence, largely genetic that it shares some abnormalities with carcinoma in situ and therefore likely represents a precursor lesion. ${ }^{26}$ It is most often diagnosed in the context of known urothelial neoplasia. Histologically, there is some architectural distortion. The nuclei are 
Table 3 Morphologic patterns of carcinoma in situ

Small cell carcinoma in situ

Large cell carcinoma in situ

Denuding carcinoma in situ 'denuding cystitis'

Undermining growth

Pagetoid carcinoma in situ

Carcinoma in situ with glandular differentiation

Carcinoma in situ with squamous differentiation

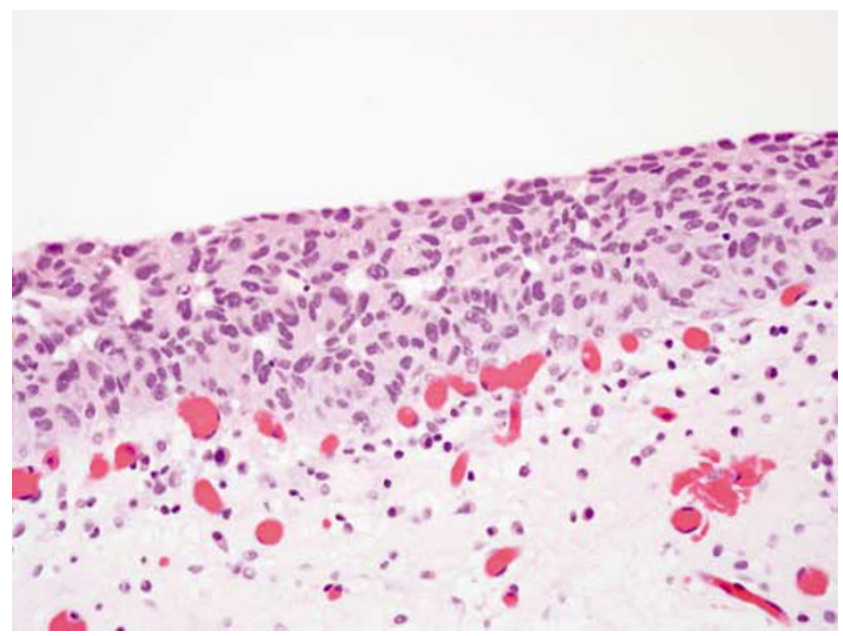

Figure 4 Urothelial carcinoma in situ. There is moderate nuclear enlargement and pleomorphism with a completely haphazard arrangement of the cells within the urothelium. A lesion similar to this may have been classified as moderate to severe dysplasia in the past but now it is included in the carcinoma in situ category.

irregularly enlarged with some hyperchromasia and pleomorphism present. Overall, the features are those of a neoplastic atypia but fall short of the criteria for carcinoma in situ outlined below. In many instances, the features resemble those of the urothelium present in low-grade papillary carcinoma (Figure 3). The current system does not grade urothelial dysplasia. Studies that have applied the $1998 \mathrm{WHO} / \mathrm{ISUP}$ criteria indicated a $15-19 \%$ risk of developing cancer with a mean follow-up of 4.9-8.2 years. $^{24,27}$

\section{Carcinoma In Situ (High-grade Intra-Urothelial Neoplasia)}

The current classification recognized the need to expand the category of carcinoma in situ to include lesions that had been graded in the moderate to severe dysplasia categories in previous systems. This change reflects current practice in major institutions treating bladder cancer. It also recognizes the general trend for under diagnosis of higher grade lesions. ${ }^{15}$ There is also recognition that this is the most reproducible diagnostic category. Carcinoma in situ is accepted as a precursor of invasive carcinoma.
Table 4 Histologic features of value in evaluating flat lesions

Thickness of urothelium

Polarity of cells

Nuclear size

Nuclear crowding

Nuclear borders including notches

Nuclear chromatin distribution

Nucleoli

Mitoses

Accompanying inflammation

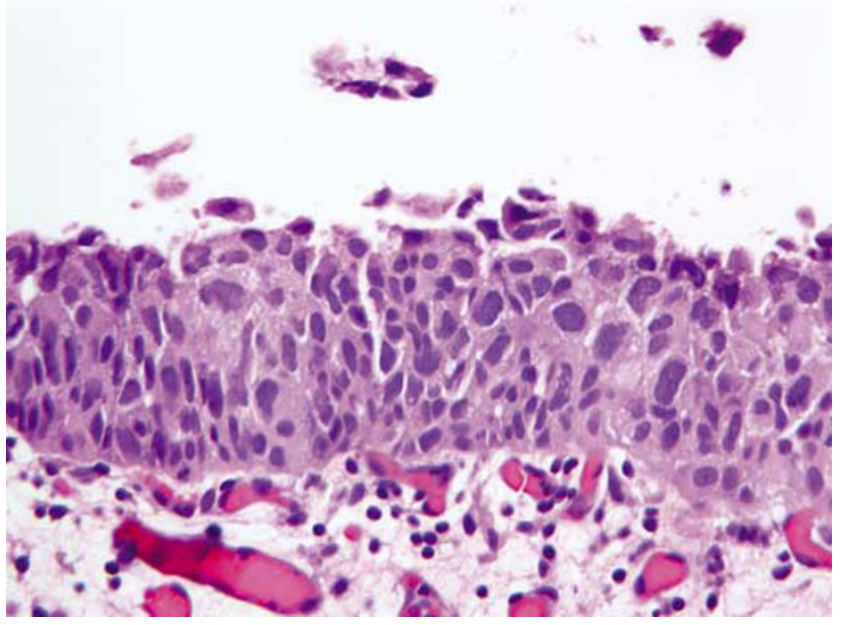

Figure 5 Urothelial carcinoma in situ. In this example, there is marked nuclear enlargement and pleomorphism. The architecture is completely disrupted. Note that many of the cells have abundant cytoplasm.

A variety of descriptive terms have been applied to carcinoma in situ (Table 3). ${ }^{14,25}$ These are helpful in highlighting the morphologic variability of the lesion, but have no clinical significance. Histologically, carcinoma in situ is characterized by architectural disorder with haphazard orientation of nuclei and nuclear crowding and clustering (Figure 4; Table 4). There is nuclear pleomorphism with variable nuclear enlargement, hyperchromasia and single to multiple nucleoli (Figure 5). The atypical cells need not involve the full thickness of the epithelium and at the minimum single malignant cells growing in a pagetoid manner are sufficient for the diagnosis. Individual cells tend to show marked cytologic atypia, but an increased nuclear to cytoplasmic ratio is not a prerequisite (not present in the large cell type of carcinoma in situ). In some cases, only a few isolated cells are present clinging to the basement membrane (denuding carcinoma in situ) (Figure 6). In other cases, the surface may be completely denuded with the carcinoma in situ present in von Brunn's nests only. This pattern should not be overdiagnosed as invasive carcinoma. 


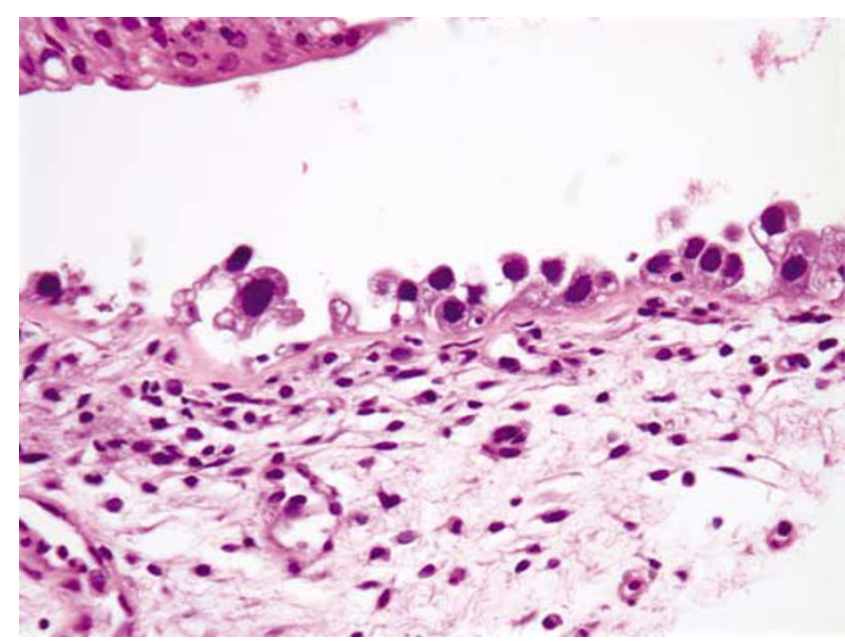

Figure 6 Urothelial carcinoma in situ. The denuding pattern is characterized by poorly cohesive cells that typically have large, pleomorphic and hyperchromatic nuclei.

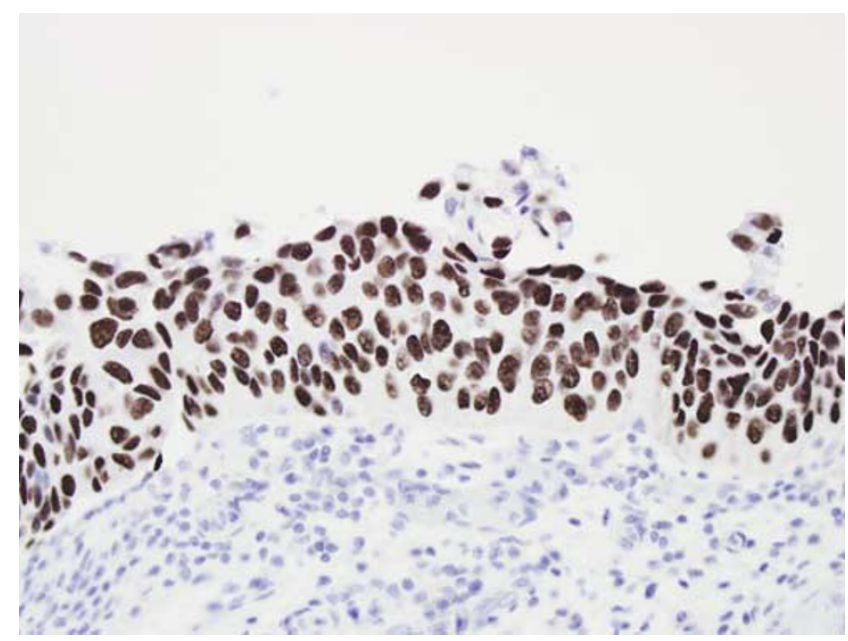

Figure 7 Urothelial carcinoma in situ. There is strong and diffuse overexpression of p53 protein.

Carcinoma in situ is most often seen in association with high-grade papillary or invasive urothelial carcinoma. De novo carcinoma in situ accounts for only $1-3 \%$ of newly diagnosed cases of bladder cancer. ${ }^{28,29}$ These patients are at significant risk for the development of invasive carcinoma. In contemporary series, progression to invasive carcinoma occurs in up to $25 \%$ of patients and the cancerspecific survival is in the $75-85 \%$ range. ${ }^{30-33}$

\section{Value of Immunohistochemistry in Flat Lesions}

A variety of immunohistochemical markers have been studied as adjuncts to the diagnosis of flat lesions. Most cases of carcinoma in situ show diffuse and intense nuclear expression of p53 (Figure 7) and diffuse reactivity for cytokeratin 20

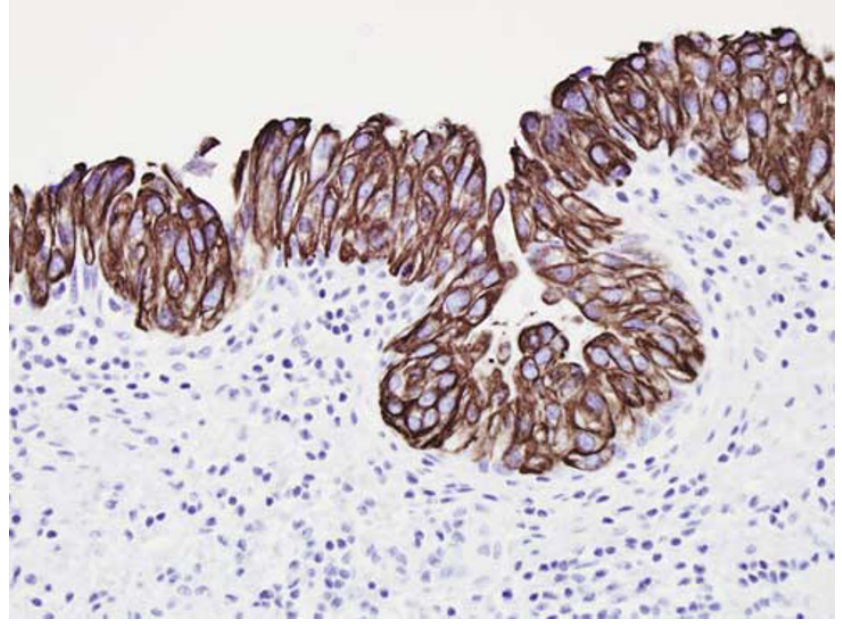

Figure 8 Urothelial carcinoma in situ. There is strong full thickness expression of cytokeratin 20 .

throughout the full thickness of the urothelium (Figure 8). ${ }^{34,35}$ In normal or reactive urothelium, p53 is generally expressed by only a few cells and with weak intensity, whereas cytokeratin 20 expression is limited to the umbrella cell layer. In my experience, the results for both are often equivocal in difficult cases and do not contribute to a final diagnosis. Proliferation is significantly higher than in reactive atypia but there is sufficient overlap to make this a difficult feature to use in individual cases. ${ }^{35}$ Other markers that have been applied to this question include CD44 and p16 but there is less experience with both. ${ }^{34,36}$

\section{Papillary lesions}

In the majority of cases, the diagnosis of a lesion as being 'papillary' in the urinary bladder is straightforward and the features to be determined are related to proper classification (grading) and evaluation of stage. Occasionally there can be problems in differential diagnosis with lesions that can mimic a papillary process (polypoid or papillary cystitis, fragmented or tangentially cut flat lesions) or papillary lesions that are not, strictly speaking, urothelial (nephrogenic adenoma and condyloma acuminatum).37,38

More problematic is the concept of papillary hyperplasia and when to call a small lesion a true papillary neoplasm particularly in the setting of known papillary neoplasia. If a true papillary stalk is evident, then the lesion is graded and classified into the categories described below. A papillary stalk is defined by the presence of a central fibrovascular core in an exophytic lesion. In the presence of known neoplasia, I am quite aggressive in diagnosing a lesion as a papillary tumor.

For cases with an undulating surface or tenting of the urothelium where no fibrovascular core is evident, the term papillary hyperplasia has been 


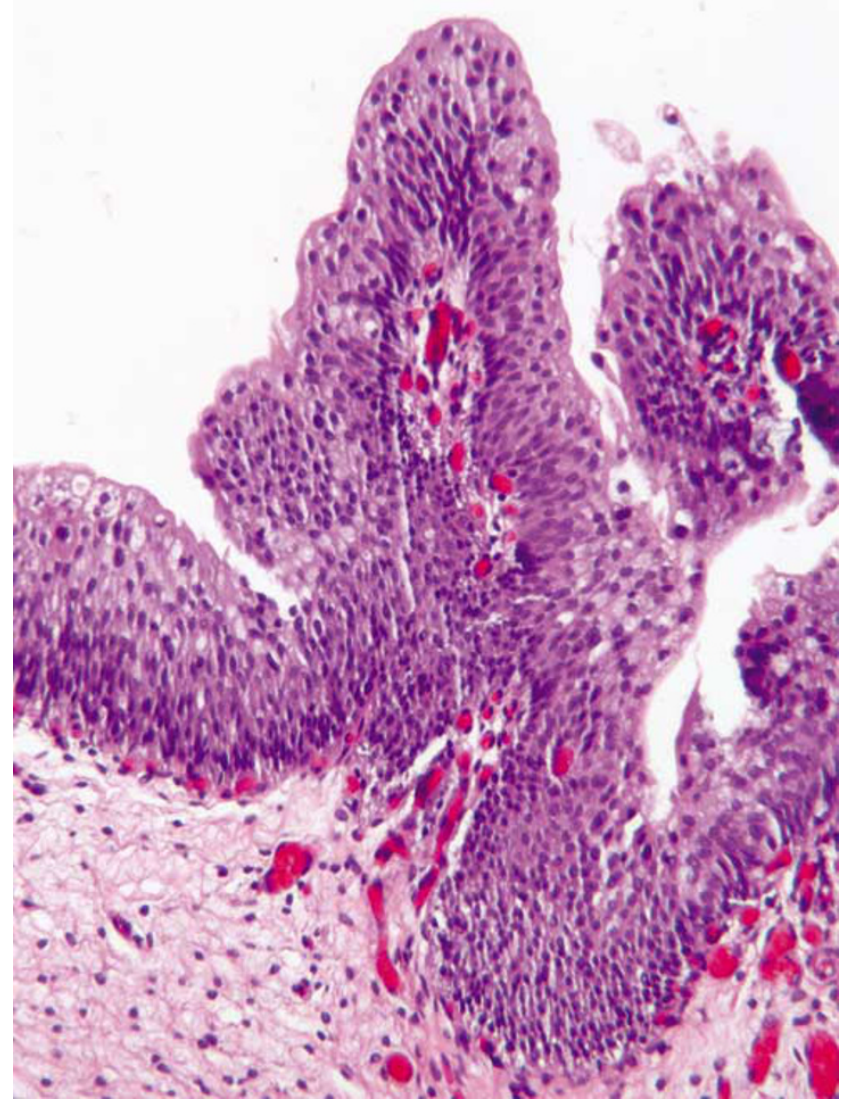

Figure 9 Urothelial papilloma. The entire lesion consists of one or two papillary fronds covered by an essentially normal urothelium.

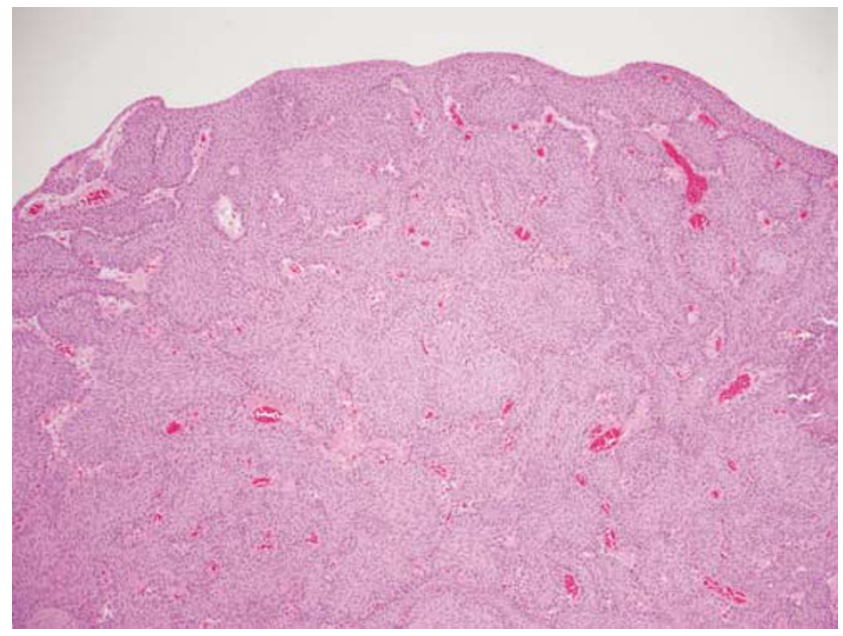

Figure 10 Inverted urothelial papilloma. At low magnification, the smooth polypoid growth and complex anastomosing trabeculae are evident.

suggested. ${ }^{39}$ In some instances, small capillaries are present in the stroma at the base of the pseudopapillae but these do not extend upwards into the stroma. These are therefore more 'pseudo-papillary' than truly papillary and that is the terminology I use

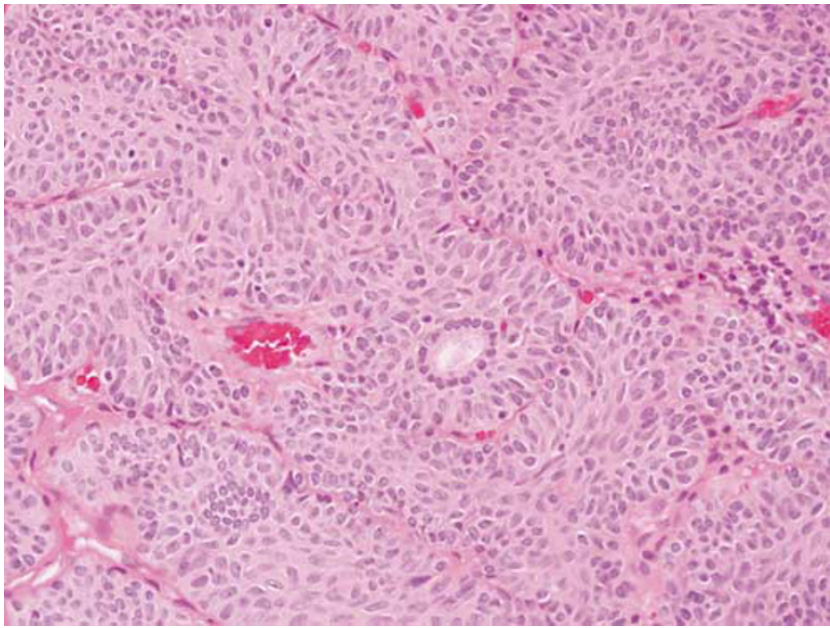

Figure 11 Inverted urothelial papilloma. Glandular differentiation is present in this lesion. Note the tendency of the basilar nuclei to line up perpendicular to the basement membrane.

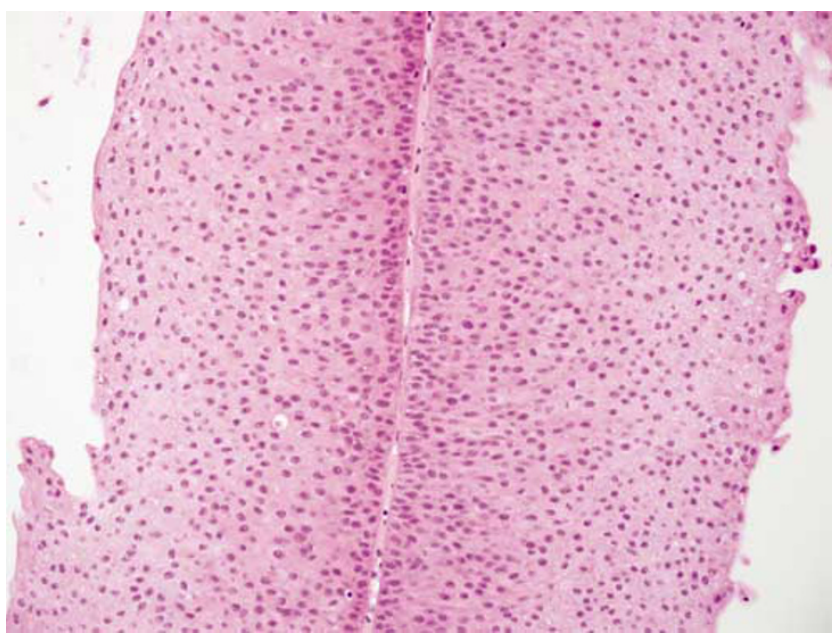

Figure 12 Papillary urothelial neoplasm of low malignant potential. The urothelium is thickened with only minimal cytologic and architectural atypia.

in my practice. The diagnosis in specimens with a pseudo-papillary architecture is dependent on evaluating the surface urothelium as a 'flat' lesion; if unequivocal dysplasia or carcinoma in situ is present, the diagnosis should be dysplasia or carcinoma in situ.

\section{Urothelial Papilloma}

There has been a long-standing controversy regarding the nature of papillary lesions with minimal cytologic atypia. ${ }^{40}$ An early definition by Mostofi ${ }^{41}$ restricted the term papilloma to non-invasive papillary lesions covered by urothelium that was indistinguishable from normal urothelium. This definition was adopted in the WHO (1973) classification. ${ }^{1}$ The use of this term by some experts for up to one-third of all papillary lesions was a major 
Table 5 Papillary urothelial neoplasms: architectural features

\begin{tabular}{llll}
\hline & PUNLMP & Low grade & High grade \\
\hline Papillae & $\begin{array}{l}\text { Delicate } \\
\text { Rarely fused }\end{array}$ & $\begin{array}{l}\text { Delicate } \\
\text { Occasionally fused }\end{array}$ & $\begin{array}{l}\text { Delicate } \\
\text { Fused and branching }\end{array}$ \\
& Polarity normal & $\begin{array}{l}\text { Predominantly ordered with minimal } \\
\text { crowding and loss of polarity } \\
\text { Anganization }\end{array}$ & $\begin{array}{l}\text { Predominantly disordered with crowding, } \\
\text { overlapping cells and loss of polarity } \\
\end{array}$ \\
& $\begin{array}{l}\text { Any thickness } \\
\text { Most increased } \\
\text { Cohesive cells }\end{array}$ & $\begin{array}{l}\text { Most increased } \\
\text { Cohesive cells }\end{array}$ & Any thickness-may be single cells (denuding) \\
& & Often discohesive
\end{tabular}

${ }^{\mathrm{a}}$ Papillary urothelial neoplasm of low malignant potential.

Table 6 Papillary urothelial neoplasms: nuclear features

\begin{tabular}{llll}
\hline & PUNLMPa & Low grade & High grade \\
\hline Size & Mildly enlarged & Enlarged & Enlarged \\
& Uniform & Some variation & Marked variability \\
Shape & Elongated & Elongated_oval_round & Pleomorphic \\
& Uniform & Slight variation & Frequently coarse with marked variation \\
Chromatin & Fine & Fine with some variation & Prominent \\
Nucleoli & Absent to inconspicuous & Usually inconspicuous & Single to multiple \\
& & & Frequent \\
Mitoses & Rare & Infrequent & Any level
\end{tabular}

${ }^{\mathrm{a}}$ Papillary urothelial neoplasm of low malignant potential.

stimulant to the reevaluation of these lesions that began in 1997., ${ }^{2,3}$ The current classification retains the restrictive traditional WHO criteria. ${ }^{1,13,14}$ Histologically, papilloma is characterized by fine papillary fronds without fusion or complexity. Individual fronds are covered by an essentially normal urothelium without architectural or cytologic atypia (Figure 9). The absolute number of cell layers is not a criterion for diagnosis but the urothelium should not be thickened.

Lesions meeting these restricted criteria occur at a younger age than other urothelial bladder tumors and often present with only one or a few papillary processes. Using the restrictive criteria recommended, these lesions account for approximately $1 \%$ of papillary tumors. ${ }^{40}$ They have a low recurrence rate with a low risk for the subsequent development of higher grade tumors. ${ }^{40,42,43}$

\section{Inverted Urothelial Papilloma}

Inverted urothelial papilloma is a distinct clinical pathologic entity that occurs over a wide age range. These develop throughout the urinary tract but are most common in the trigone region. In rare cases, they can be multifocal. ${ }^{44}$ Inverted papilloma is associated with a low risk of recurrence $(<5 \%)$, distinctly different from papillary urothelial neoplasms. ${ }^{44,45}$ Recent genetic data support the concept that these are not related to papillary urothelial neoplasms. ${ }^{46}$ Cases of synchronous inverted papilloma and papillary carcinoma are well described though, in my experience, extraordinarily rare; distinction from papillary carcinoma with an inverted growth pattern can be problematic. $^{46,47}$

By cystoscopy, these lesions typically have an exophytic polypoid growth pattern and can be pedunculated. Histologically, inverted papilloma consists of anastomosing trabeculae of urothelium covered by a normal or attenuated urothelium (Figure 10). Multiple sites of origin from the surface urothelium are usually present. The basal layer is often prominent with the basilar nuclei lined up perpendicular to the basement membrane. The cells in the central part of the trabeculae can be spindled with a streaming growth pattern. In general, there is no significant nuclear pleomorphism, although occasionally mild atypia can be present. ${ }^{48}$ Mitotic figures are rare or absent. Squamous or glandular differentiation may be present (Figure 11). In transurethral resection material, the fragmentation of the lesion may result in apparent papillary structures making diagnosis difficult.

\section{Papillary Urothelial Neoplasm of Low Malignant Potential}

The creation of this category represented a compromise between the 'papilloma' camp and those insisting on the use of 'carcinoma' for all papillary lesions. The 1998 consensus statement acknowl- 


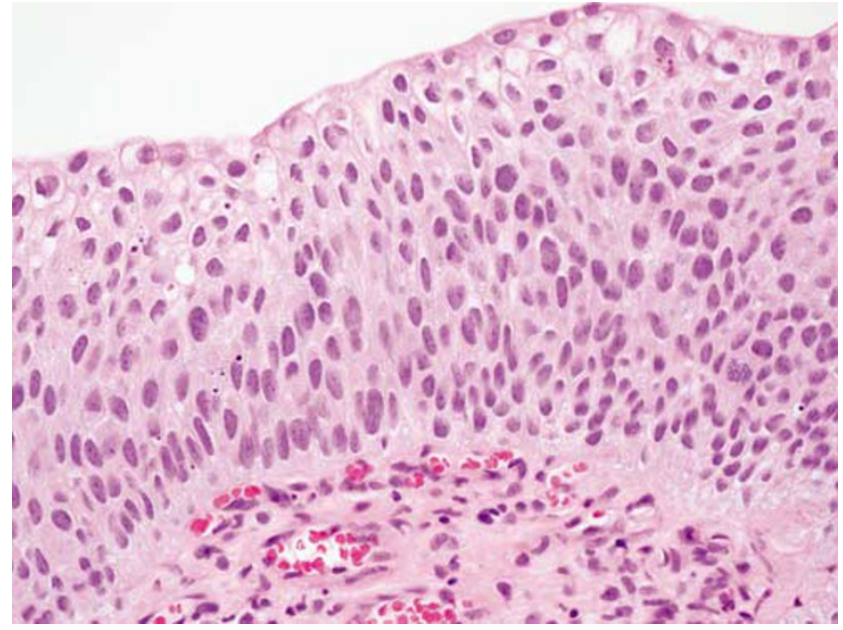

Figure 13 Low-grade papillary urothelial carcinoma. The urothelium is thickened with the architecture generally intact but with some irregularity in the nuclear spacing. There is variability in the nuclear size with variable degrees of hyperchromasia.

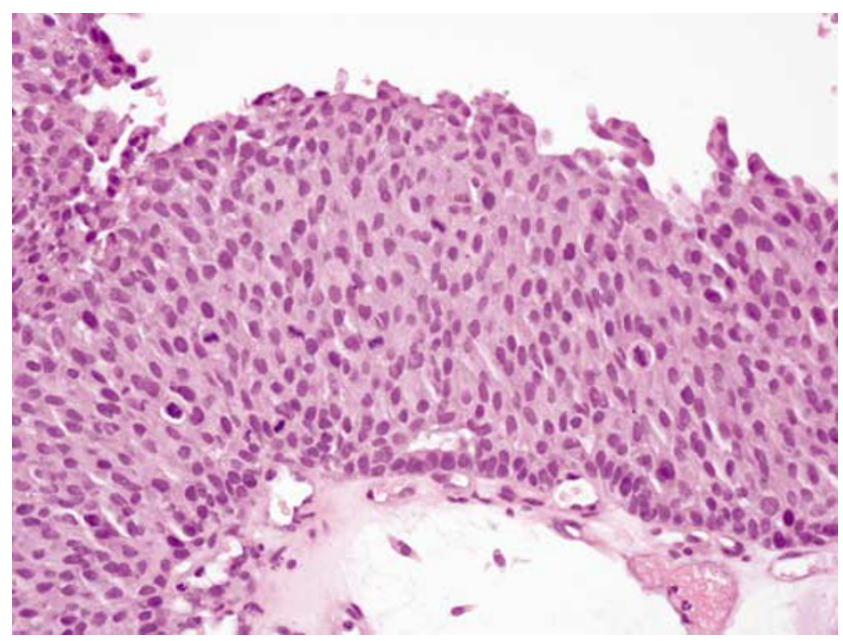

Figure 14 High-grade papillary urothelial carcinoma. The urothelium has increasing architectural irregularity (compare with Figure 13) and mild to moderate cytologic atypia. There are numerous mitotic figures scattered throughout the urothelium.

edged that the lower grade papillary neoplasms were not intrinsically malignant but were associated with a significant risk for the development of new papillary tumors (recurrence). These lesions at the lower end of the spectrum were accepted as clinically significant with close clinical follow-up necessary but further intravesical therapy not indicated. Morphologically, this category largely though not completely corresponds to grade 1 papillary carcinoma in the 1973 WHO system. $^{1}$ The tumor consists of delicate papillae with little or no fusion. The covering urothelium is usually thickened but shows minimal, if any, architectural irregularity (Figure 12). Nuclei are normal in size to slightly enlarged and lack significant nuclear hyperchromasia or pleomorphism. The chromatin

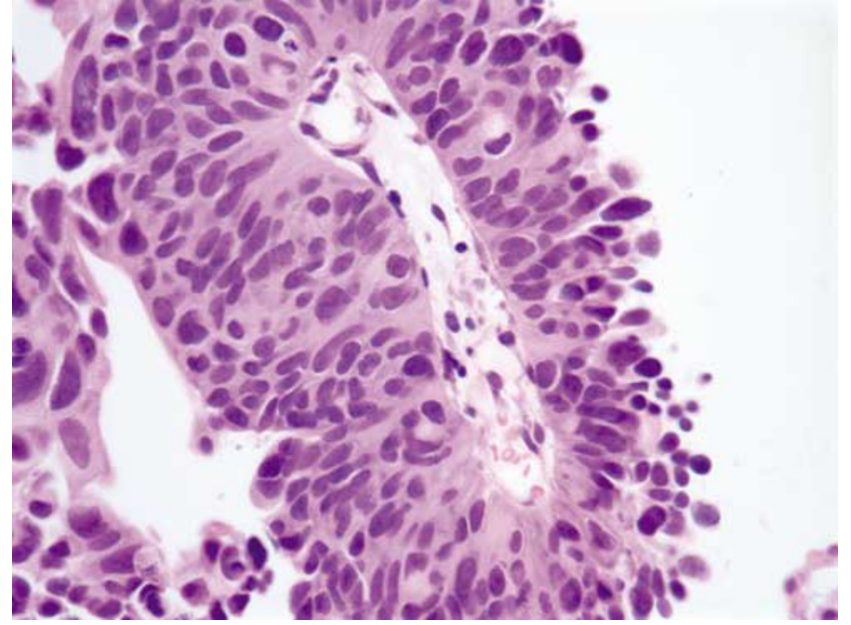

Figure 15 High-grade papillary urothelial carcinoma. There is significant disturbance of the architecture and the cells have pleomorphic and hyperchromatic nuclei. In this example, there is a tendency of the cells to be discohesive.

is fine and nucleoli are inconspicuous. Mitoses are infrequent and basally located when present (Tables 5 and 6).

These tumors have a significantly lower rate of recurrence than either low- or high-grade papillary carcinomas and have a very low rate of stage progression. $^{8,17,49-52}$ In a review of published studies, the mean tumor recurrence rate was found to be $36 \%$ and the stage progression rate to be $3.7 \% .^{12}$

\section{Papillary Urothelial Carcinoma, Low-Grade}

This category contains the intermediate group of lesions. In the 1973 WHO system, this would include roughly the lower one-half to two-thirds of grade 2 papillary carcinoma. ${ }^{16,17}$ The papillae are largely delicate and separate but some fusion may be seen. At low magnification, there is a generally ordered appearance to the cells within the epithelium (Figure 13). The nuclei tend to be uniformly enlarged and retain the elongated to oval shape of normal urothelial cells. The chromatin remains fine with small and generally inconspicuous nucleoli. Mitoses may be present but are few and generally remain basally located (Tables 5 and 6).

These tumors have a significantly higher recurrence rate than for papillary urothelial neoplasm of low malignant potential and similar to high-grade papillary carcinomas. ${ }^{8}$ They also have a higher rate of stage progression than papillary urothelial neoplasm of low malignant potential but significantly lower than for high-grade papillary carcinoma. ${ }^{8,18,51}$ Low-grade tumors can be invasive though this is distinctly uncommon. A review of the literature revealed a mean recurrence rate of $50 \%$ and mean stage progression rate of $10 \% .{ }^{12}$ Patients with these tumors require close clinical follow-up, though 
recently it has been suggested that this can be less frequent than for patients with high-grade tumors. ${ }^{20}$ A single dose of intravesical therapy is optional but maintenance intravesical therapy is not recommended. ${ }^{22}$

\section{Papillary Urothelial Carcinoma, High-Grade}

As discussed above, many WHO grade 2 (1973) tumors (roughly the upper one-half to one-third) have a significant frequency of invasion and biologically have more in common with the grade 3 tumors. These tumors not only have a significant risk of recurrence but also have a substantial risk of invasion and progression to muscle invasive disease. For this reason, the consensus was that these were better included in a high-grade category with the traditional WHO grade 3 neoplasms.

The papillae are frequently fused forming apparent solid masses. The overall impression is one of disordered growth (Figure 14). The epithelium is of variable thickness and may resemble 'denuding carcinoma in situ' in some instances. Individual cells are haphazardly arranged within the epithelium and have a generally discohesive nature. Nuclei are hyperchromatic and pleomorphic (Figure 15). The chromatin is dense, irregularly distributed and often clumped. Nucleoli may be single or multiple and are often prominent. Mitoses are generally frequent and may be seen at any level of the epithelium (Tables 5 and 6). Several studies have looked at a variety of biologic markers in papillary tumors and their relationship to the three groups; for the most part, these have demonstrated significant differences of the respective marker in the different categories. ${ }^{17,53,54}$

The overall progression rate (to invasive carcinoma) ranges from 15 to $40 \%$. For high-grade pTa tumors, intravesical bacillus Calmette-Guérin therapy with an induction course and maintenance is recommended. ${ }^{22}$ Heterogeneity of grade is recognized in papillary lesions ${ }^{55}$ and the consensus is that tumors should be graded on their worst part, although this needs further study. ${ }^{7,13}$

\section{Disclosure/conflict of interest}

The author declares no conflict of interest.

\section{References}

1 Mostofi FK, Sobin LH. Histologic Typing of Urinary Bladder Tumors. World Health Organization: Geneva, 1973.

2 Murphy WM, Beckwith JB, Farrow GM. Atlas of Tumor Pathology: Tumors of the Kidney, Bladder, and related Urinary Structures. Armed Forces Institute of Pathology 3rd series fascicle American Registry of Pathology: Washington, DC, 1994.
3 Jordan AM, Weingarten J, Murphy WM. Transitional neoplasms of the urinary bladder, can biological potential be predicted from histological grading. Cancer 1987;60:2766-2774.

4 Witjes JA, Moonen PMJ, van der Heijden AG. Review pathology in a diagnostic bladder cancer trial: effect on patient risk category. Urology 2006;67:751-755.

5 Kiemeney LALM, Witjes JA, Verbeek ALM, et al. The clinical epidemiology of superficial bladder cancer. $\mathrm{Br}$ J Cancer 1993;67:806-812.

6 Bostwick DG, Mikuz G. Urothelial papillary (exophytic) neoplasms. Virch Arch 2002;441:109-116.

7 Epstein JI, Amin MB, Reuter VR, et al. The World Health Organization/International Society of Urologic Pathology consensus classification of urothelial (transitional) neoplasms of the urinary bladder. Am J Surg Pathol 1998;22:1435-1448.

8 Malmström PU, Busch C, Norlen BJ. Recurrence, progression and survival in bladder cancer: a retrospective analysis of 232 patients with greater than or equal to 5-year follow-up. Scand J Urol Nephrol 1987;21:185-195.

9 Mostofi FK, Davis Jr CJ, Sesterhenn IA. Histological Typing of Urinary Bladder Tumors: International Histological Classification of Tumors. World Health Organization: Geneva, 1999.

10 Cheng L, Bostwick DG. World Health Organization and International Society of Urologic Pathology Classification and a two-number grading system of bladder tumors. Cancer 2000;88:1513-1516.

11 Oyasu R. World Health Organization and International Society of Urological Pathology Classification and twonumber grading system of bladder tumors. Cancer 2000;88:1509-1512.

12 Lopez-Beltran A, Montironi R. Non-invasive urothelial neoplasms: according to the most recent WHO classification. Eur Urol 2004;46:170-176.

13 Eble JN, Epstein JI, Sauter G, et al. World Health Organization Histologic and Genetic Typing of Tumors of the Kidney, Urinary Bladder, Prostate Gland and Testis. IARC Press: Lyon, 2004.

14 Murphy WM, Grignon DJ, Perlman E. Atlas of Tumor Pathology: Tumors of the Kidney, Bladder, and related Urinary Structures. Armed Forces Institute of Pathology 4th series fascicle American Registry of Pathology: Washington, DC, 2004.

15 Reuter VE. The pathology of bladder cancer. Urology 2006;67(Suppl 3A):11-18.

16 Samaratunga H, Makarov DV, Epstein JI. Comparison of WHO/ISUP and WHO classification of noninvasive papillary urothelial neoplasms for risk of progression. Urology 2002;60:315-319.

17 Yin H, Leong AS-Y. Histologic grading of noninvasive papillary urothelial tumors: validation of the 1998 WHO/ISUP system by immunophenotyping and follow up. Am J Clin Pathol 2004;121:679-687.

18 Herr HW, Donat SM, Reuter VE. Management of low grade papillary bladder tumors. J Urol 2007;178:1201-1205.

19 Burger M, van der Aa MNM, van Oers JMM, et al. Prediction of progression on non-muscle invasive bladder cancer by WHO 1973 and 2004 grading and by FGFR3 mutation status: a prospective study. Eur Urol 2008;54:835-844.

20 Herr HW. Low risk bladder tumors-less is more. J Urol 2008;179:13-14.

21 Nieder AM, Soloway MS. Eliminate the term 'superficial’ bladder cancer. J Urol 2006;175:417-418. 
22 Hall MC, Chang SS, Dalbagni G, et al. Guideline for the management of non muscle invasive bladder cancer (stages Ta, T1, and Tis): 2007 update. J Urol 2007;178:2314-2330.

23 Amin MB, Srigley JR, Grignon DJ, et al. Urinary Bladder, ureter, and renal pelvis: protocol applies to all carcinomas of the urinary bladder, ureter and renal pelvis. Arch Pathol Lab Med 2003;127: 1263-1279 (Protocol revision date, January 2005; CAP Web page:http://www.cap.org/apps/docs/cancer_ protocols/2005/urbladder05_pw.pdf).

24 Cheng L, Cheville JC, Neumann RM, et al. Flat intraepithelial lesions of the urinary bladder. Cancer 2000;88:625-631.

25 Murphy WM, Busch C, Algaba F. Intraepithelial lesions of urinary bladder: morphologic considerations. Scand J Urol Nephrol Suppl 2000;205:67-81.

26 Obermann EC, Junker K, Stoehr R, et al. Frequent genetic alterations in flat urothelial hyperplasia and concomitant papillary bladder cancer as detected by CGH, LOH, and FISH analysis. J Pathol 2003;199: 50-57.

27 Cheng L, Cheville JC, Neumann RM, et al. Natural history of urothelial dysplasia of the bladder. Am J Surg Pathol 1999;23:443-447.

28 Farrow G. Pathology of carcinoma in situ of the urinary bladder and related lesions. J Cell Biochem 1992;161:39-43.

29 Orozco R, Martin A, Murphy W. Carcinoma in situ of the urinary bladder: clues to host involvement in human carcinogenesis. Cancer 1994;74:115-122.

30 Melamed M, Voutsa N, Grabstald H. Natural history and clinical behavior of in situ carcinoma of the human urinary bladder. Cancer 1964;17:1533-1545.

31 Cheng L, Cheville JC, Neumann RM, et al. Survival of patients with carcinoma in situ of the urinary bladder. Cancer 1999;85:2469-2474.

32 Gofrit ON, Pode D, Pizov G, et al. The natural history of bladder carcinoma in situ after an initial response to bacillus Calmette-Guerin immunotherapy. Urol Oncol 2008; e-pub ahead of print.

33 Sylvester RJ, van der Meijden A, Witjes JA, et al. Highgrade Ta urothelial carcinoma and carcinoma in situ of the bladder. Urology 2005;66(6 Suppl 1):90-107.

34 McKenney JK, Amin MB. The role of immunohistochemistry in the diagnosis of urinary bladder neoplasms. Semin Diagn Pathol 2005;22:69-87.

35 Yin $\mathrm{H}, \mathrm{He} \mathrm{Q}, \mathrm{Li} \mathrm{T}$, et al. Cytokeratin 20 and Ki-67 to distinguish carcinoma in situ from flat non-neoplastic urothelium. Appl Immunohistochem Mol Morphol 2006;14:260-265.

36 Yin M, Bastacky S, Parwani AV, et al. p16(ink4) immunoreactivity is a reliable marker for urothelial carcinoma in situ. Hum Pathol 2008;39:527-535.

37 Cheng L, Bostwick DG. Overdiagnosis of bladder carcinoma. Anal Quant Cytol Histol 2008;30:261-264.

38 Jones EC. Urinary bladder. Mimics of neoplasia and new pathologic entities. Urol Clin North Am 1999;26:509-534.

39 Taylor DC, Bhagavan BS, Larsen MP, et al. Papillary urothelial hyperplasia: a precursor to papillary neoplasm. Am J Surg Pathol 1996;20:1481-1488.

40 Eble JN, Young RH. Benign and low grade papillary lesions of the urinary bladder: a review of the papilloma-papillary carcinoma controversy and a report of 5 typical papillomas. Semin Diag Pathol 1987;6:351-371.

41 Mostofi FK. Pathological aspects and spread of carcinoma of the bladder. JAMA 1968;206:1764-1771.

42 Cheng L, Darson M, Cheville JC, et al. Urothelial papilloma of the bladder: clinical and biologic implications. Cancer 1999;86:2098-2101.

43 McKenney JK, Amin MB, Young RH. Urothelial (transitional cell) papilloma of the urinary bladder: a clinicopathologic study of 26 cases. Mod Pathol 2003;16:623-629.

44 Sung M-T, MacLennan GT, Lopez-Beltran A, et al. Natural history of urothelial inverted papilloma. Cancer 2006;107:2622-2627.

45 Cheng CW, Chan LW, Chan CK, et al. Is surveillance necessary for inverted papilloma in the urinary bladder and urethra? ANZ J Surg 2005;75:213-217.

46 Jones TD, Zhang S, Lopez-Beltran A, et al. Urothelial carcinoma with an inverted growth pattern can be distinguished from inverted papilloma by fluorescence in situ hybridization immunohistochemistry and morphologic analysis. Am J Surg Pathol 2007;31:1861-1867.

47 Amin MB, Gomez JA, Young RH. Urothelial transitional cell carcinoma with endophytic growth patterns: a discussion of patterns of invasion and problems associated with assessment of invasion in 18 cases. Am J Surg Pathol 1997;21:1057-1068.

48 Broussard JN, Tan PH, Epstein JI. Atypia in inverted urothelial papillomas: pathology and prognostic significance. Hum Pathol 2004;35:1499-1504.

49 Cheng L, Neumann RL, Bostwick DG. Papillary urothelial neoplasms of low malignant potential: clinical and biological implications. Cancer 1999;86:2102-2108.

50 Holmang S, Andius $\mathrm{P}$, Hedelin $\mathrm{H}$, et al. Stage progression in Ta papillary urothelial tumors: relationship to grade, immunohistochemical expression of tumor markers, mitotic frequency and DNA ploidy. J Urol 2001;165:1124-1128.

51 Oosterhuis JW, Schapers RF, Janssen-Heijnen ML, et al. Histological grading of papillary urothelial carcinoma of the bladder: prognostic value of the $1998 \mathrm{WHO} /$ ISUP classification system and comparison with conventional grading systems. J Clin Pathol 2002;55:900-905.

52 Campbell PA, Conrad RJ, Campbell CM, et al. Papillary urothelial neoplasm of low malignant potential: reliability of diagnosis and outcome. BJU Int 2004;93:1228-1291.

53 Cheng L, Neumann RM, Nehra A, et al. Cancer heterogeneity and its biological implications in the grading of urothelial carcinoma. Cancer 2000;88:1663-1670.

54 Cina SJ, Lancaster-Weiss KJ, Lecksell K, et al. Correlation of Ki67 and p53 with the new World Health Organization/International Society of Urological Pathology classification system for urothelial neoplasms. Arch Pathol Lab Med 2001;125:646-651.

55 Pich A, Chiusa L, Formiconi A, et al. Biological differences between noninvasive papillary urothelial neoplasms of low malignant potential and low-grade (grade 1) papillary carcinoma of the bladder. Am J Surg Pathol 2001;25:1528-1533. 